\title{
Sulfasalazine-Induced Pancytopenia Indicative of Bone Marrow Suppression: A Case Report
}

\author{
Abdulrahman S Alanazi
}

\begin{abstract}
Ulcerative colitis (UC) is a chronic disease characterized by the inflammation and ulceration of the colon mucosa. The present case report of pancytopenia associated with sulfasalazine treatment substantiates a few other published reports and further our knowledge of sulfasalazine tolerability. We report a case of sulfasalazine-induced pancytopenia indicative of bone marrow suppression in an 18 years old Saudi girl with UC. After two months of treatment with azathioprine, sulfasalazine and prednisolone, her hematological indicators declined to a greater extent. Initially, all medications except sulfasalazine were stopped but hematology did not improve so that sulfasalazine was also discontinued, but prednisolone was used for the next 30 days. Hematological indicators of the patient were improved and sulfasalazine was restarted at a dose of $500 \mathrm{mg}$ t.i.d. However, within a month, hematological indicators declined drastically, which led to discontinuation of sulfasalazine re-treatment. The case of sulfasalazine-induced pancytopenia presented herein is suggestive of bone marrow suppression keeping in view the previous reports of UC patients regarding the development of sulfasalazine-induced bone marrow suppression and associated pancytopenia.
\end{abstract}

Keywords: Sulfasalazine; Ulcerative colitis; Pancytopenia; Bone marrow suppression

\section{Introduction}

Ulcerative colitis (UC) is a chronic disease characterized by the inflammation and ulceration of the colon mucosa. Aminosalicylates are the first line medication for UC among which sulfasalazine is a most effective drug for the induction

\footnotetext{
Manuscript accepted for publication March 31, 2014

Unaizah College of Pharmacy, Qassim University, Saudi Arabia. Email: Alanazi_abdulrahman@yahoo.com
}

doi: http://dx.doi.org/10.14740/jmc1767w of $\mathrm{UC}$ remission as well as for the maintenance of remission. Although the sulfasalazine therapy is associated with side effects, including bone marrow suppression [1], its long-term use is evident from the follow-up studies [2]. Therapies for UC may also include one or more modalities among steroids, antibiotics, immunomodulators, nicotine, heparin, probiotics, and short-chain fatty acids, and curative surgery which may become necessary for patients unresponsive to aminosalicylates or develop steroid dependency [3, 4].

There are several reports of one or more aspects of sulfasalazine-associated bone marrow suppression such as pancytopenia, thrombocytopenia, hemophagocytosis, agranulocytosis, erythroid hypoplasia and bone marrow necrosis in UC patients. Pertinent of these cases are; a fatal case of sulfasalazine-associated agranulocytosis of a 39 years old man [5]; a 22 years old Caribbean man with UC who was being treated with $3 \mathrm{~g} /$ day sulfasalazine daily exhibited bone marrow necrosis in biopsies within 4 - 5 weeks of treatment and discontinuity of sulfasalazine led to his complete recovery [6]; a 26 years old man with slow acetylator phenotype, who had been treated with sulfasalazine ( $2 \mathrm{~g}$ /day) for three years, developed extensive oral ulceration upon increasing dose to $4 \mathrm{~g} /$ day. Among the pathologies, bone marrow examination showed severe hematopoietic megaloblastic changes. The patient recovered upon a strategic administration of folinic acid, hydroxycobalamin and folic acid following sulfasalazine discontinuation [7]; a 79 years old woman developed sulfasalazine-associated agranulocytosis seven weeks after treatment and recovered upon sulfasalazine discontinuation [8]; a 32 years old Korean man after two years of sulfasalazine treatment for UC control developed pancytopenia and hemophagocytosis in bone marrow [9]; and a 52 years old Korean woman with cytomegalovirus infection developed hemophagocytosis in the bone marrow [10].

In this article, a case of a female patient who developed signs of bone marrow suppression associated with sulfasalazine treatment has been presented.

\section{Case Report}

A Saudi girl (age: 18 years old, body weight: $70 \mathrm{~kg}$, height: 


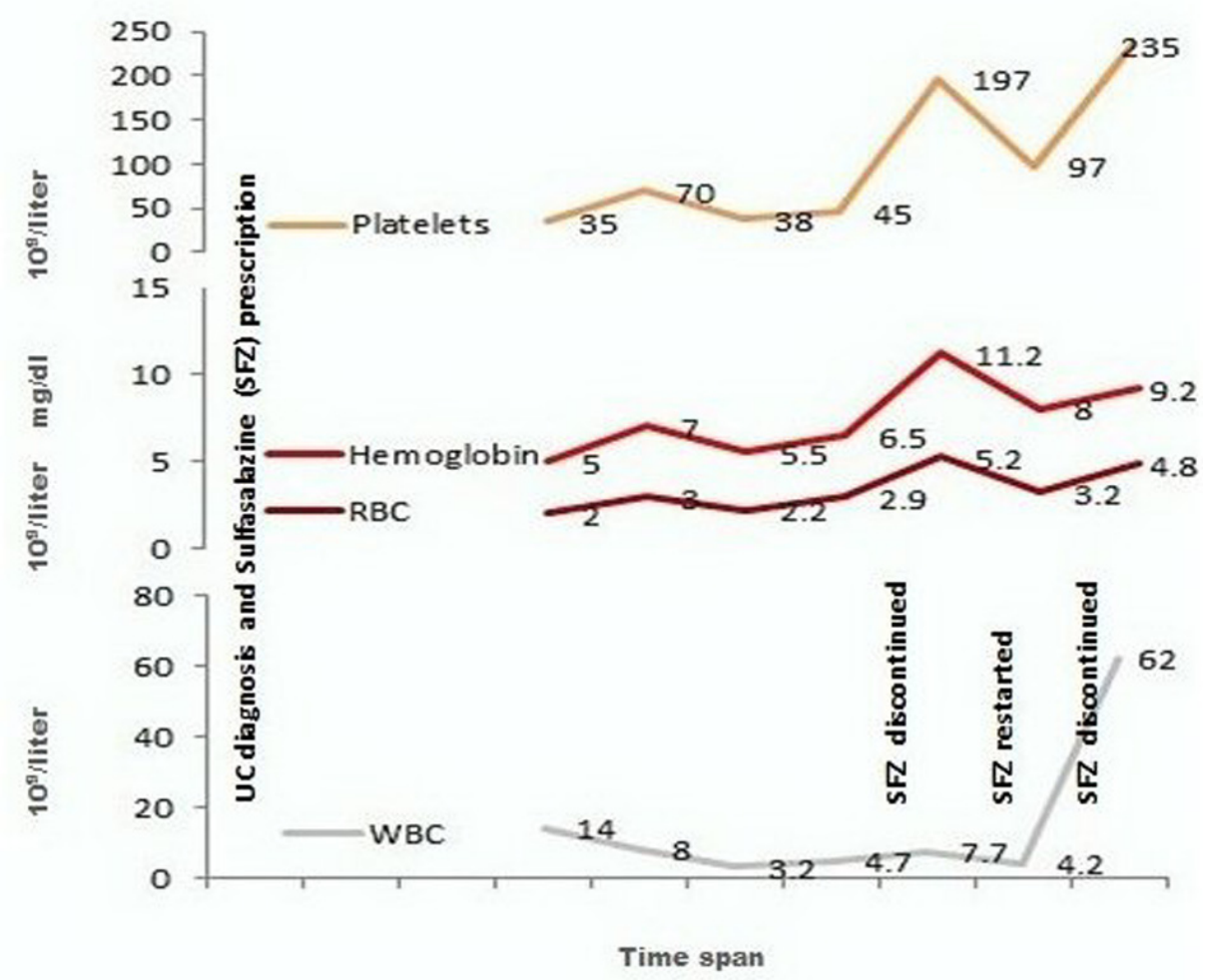

Figure 1. Changes in the hematological indicators of the patient against interventions.

$165 \mathrm{~cm}$ ) was diagnosed as UC in July 2012 based on clinical presentation (gastrointestinal bleeding, diarrhea, abdominal pain, and so on) and colonoscopy which was subsequently confirmed by the specialized healthcare expertise of the institution. She was prescribed azathioprine $50 \mathrm{mg}$, b.i.d., sulfasalazine $1 \mathrm{~g}$, t.i.d., p.o., and prednisolone $(50 \mathrm{mg}$ for the first 30 days, $40 \mathrm{mg}$ for the next 30 days, $30 \mathrm{mg}$ for the next 30 days, $25 \mathrm{mg}$ for the next 30 days and then tapered down to discontinue over a 2-week period). During the treatment period, the patient sustained low grade fever with coughing and was treated with augmentin $1 \mathrm{~g}$, b.i.d., for seven days. Because of a persistent fever, the patient was examined two months after the start of treatment and the laboratory tests identified hematological perturbations. The patient was hospitalized and received blood/platelet transfusions. All medications were stopped except sulfasalazine. The patient was discharged within 10 days of hospitalization with better hematological picture (Fig. 1).

In mid-November, the patient who was re-admitted exhibited poor hematology, which did not improve further in the next 10 days. At this stage, all ongoing medication, including sulfasalazine, was discontinued except prednisolone (20 mg/day for the next 30 days). In the following days, hematological indicators of the patients were improved and attained even better level with the passage time. At this stage, sulfasalazine was restarted at a dose of $500 \mathrm{mg}$ t.i.d. However, within a month, hematological indicators declined drastically, which led to discontinuation of sulfasalazine retreatment (Fig. 1). The patient was not re-challenged with sulfasalazine and by the time of last follow-up, she was UC asymptomatic and was being managed with non-medical treatments.

\section{Discussion}

Generally, the immunomodulators are associated with several side effects, and sulfasalazine intolerance is also not too rare. Sulfasalazine associated pancytopenia observed in the case reported herein may underlie bone marrow suppression based on the similarity in symptoms and hematological indicators presented in the cases reviewed above [5-9] and other cases such as one pancytopenia case in a 23 years old patient with Crohn's disease [10]. Although bone marrow biopsy examinations could not be performed, on the basis of coincidence of changes in the hematological profile of the patient with changes in medication, and in the light of previous reports of strong association of pancytopenia with bone marrow cytopathology, it is reasonable to assume the case presented herein as an incidence of bone marrow suppression.

When administered orally, $25 \%$ of sulfasalazine is absorbed in the small intestine and most of it reaches the colon where bacterial activity metabolizes it into two metabolites: 
5-aminosalicylic acid and sulfapyridine. 5-aminosalicylic acid is not absorbed from the colon and manifests its therapeutic effects on the inflammatory mucosa while the sulfapyridine is absorbed from colon mucosa and is also excreted in the urine and therefore has restricted effectiveness [11]. For this sake, 5-aminosalicylic acid and sulfasalazine can be suitable alternatives and both have shown comparable efficacies in a randomized trial [12]. However, 5-aminosalicylic acid-induced pancytopenia suggestive of bone marrow suppression has also been reported [13].

Since there can be several mechanisms of bone marrow suppression such as immune reactions, infections and drug toxicity. It is recommended that sulfasalazine should be discontinued upon unexplained fever, chills, sore throat, malaise, or other nonspecific illness earlier in the treatment [8]. Some authors have also suggested liver function tests at this stage in order to explore any possibility of immune reaction $[14,15]$.

\section{Conclusion}

Sulfasalazine associated pancytopenia observed in the case reported herein is speculated as bone marrow suppression incidence by taking inferential support from previously reported cases in which pancytopenia is strongly associated with bone marrow suppression. It is suggested that symptomatic changes and laboratory profiles of the patients treated with sulfasalazine should be monitored during the first few months of treatment upon increasing dosage.

\section{Grant Support}

None.

\section{Abbreviations}

b.i.d.: bis in die (twice daily); g: gram/s; kg: kilogram/s; mg: milligram/s; p.o.: per os (orally); t.i.d.: ter in die (trice daily); UC: ulcerative colitis

\section{References}

1. Sutherland L, Macdonald JK. Oral 5-aminosalicylic acid for induction of remission in ulcerative colitis. Cochrane Database Syst Rev. 20062):CD000543.
2. Solberg IC, Lygren I, Jahnsen J, Aadland E, Hoie O, Cvancarova M, Bernklev T, et al. Clinical course during the first 10 years of ulcerative colitis: results from a population-based inception cohort (IBSEN Study). Scand J Gastroenterol. 2009;44(4):431-440.

3. Jani N, Regueiro MD. Medical therapy for ulcerative colitis. Gastroenterol Clin North Am. 2002;31(1):147166.

4. Kozuch PL, Hanauer SB. Treatment of inflammatory bowel disease: a review of medical therapy. World $\mathrm{J}$ Gastroenterol. 2008;14(3):354-377.

5. Maddocks JL, Slater DN. Toxic epidermal necrolysis, agranulocytosis and erythroid hypoplasia associated with sulphasalazine. J R Soc Med. 1980;73(8):587-588.

6. Van de Pette JE, Cunnah DT, Shallcross TM. Bone marrow necrosis after treatment with sulphasalazine. $\mathrm{Br}$ Med J (Clin Res Ed). 1984;289(6448):798.

7. Logan EC, Williamson LM, Ryrie DR. Sulphasalazine associated pancytopenia may be caused by acute folate deficiency. Gut. 1986;27(7):868-872.

8. Dery CL, Schwinghammer TL. Agranulocytosis associated with sulfasalazine. Drug Intell Clin Pharm. 1988;22(2):139-142.

9. Kim YC, Kim GM, Lee HS, Hong SI, Cheung DY, Kim JI, Park SH, et al. [A case of hemophagocytic syndrome in an ulcerative colitis patient]. Korean J Gastroenterol. 2010;56(1):45-48.

10. Mun JI, Shin SJ, Yu BH, Koo JH, Kim DH, Lee KM, Lee KJ. A case of hemophagocytic syndrome in a patient with fulminant ulcerative colitis superinfected by cytomegalovirus. Korean J Intern Med. 2013;28(3):352-355.

11. Taffet SL, Das KM. Sulfasalazine. Adverse effects and desensitization. Dig Dis Sci. 1983;28(9):833-842.

12. Rachmilewitz D. Coated mesalazine (5-aminosalicylic acid) versus sulphasalazine in the treatment of active ulcerative colitis: a randomised trial. BMJ. 1989;298(6666):82-86.

13. Kotanagi H, Ito M, Koyama K, Chiba M. Pancytopenia associated with 5-aminosalicylic acid use in a patient with Crohn's disease. J Gastroenterol. 1998;33(4):571574.

14. Lau G, Kwan C, Chong SM. The 3-week sulphasalazine syndrome strikes again. Forensic Sci Int. 2001;122(23):79-84.

15. Kampmann P, Hojlyng N, Pedersen M. [Life-threatening liver failure and severe dyscrasias in blood and lymph nodes caused by sulphasalazine]. Ugeskr Laeger. 2006;168(39):3331-3333. 